\title{
THE COMMON CAUSES LEADING TO PANCYTOPENIA AT TERTIARY CARE HOSPITAL
}

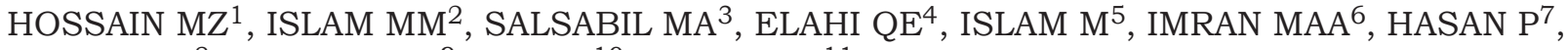 \\ RAHMAN A ${ }^{8}$, FERDOUS N ${ }^{9}$, EVA IZ $^{10}$, AZAD KAK ${ }^{11}$
}

\begin{abstract}
Pancytopenia is a triad of simultaneous presence of anemia, leucopenia and thrombocytopenia. It is result from a number of disease processes. Both hematopoietic and nonhematopoietic conditions manifest with features of pancytopenia.

Methods: This was a cross-sectional study carried out in the Department of Medicine, Dhaka Medical College Hospital between December 2016 to July 2017. We included adult patients of both sexes having age 16 years and above. Criteria for inclusion were persistent pancytopenia on peripheral blood film of more than one week duration. All patients underwent a detailed medical history and full physical examination followed by blood sampling for the investigations. After taking all the aseptic measures and with standard technique the diagnostic bone marrow aspiration and trephine biopsy were done.

Results: There were 36 patients with pancytopenia which were included in this study. There were 20 (56\%) males and 16 (44\%) females with a 1.25:1 male to female ratio and a mean age 47.30 years $\pm 15.01 \mathrm{SD}$. The most common complaints were bleeding manifestation (29/36), followed by generalized weakness (27/36) and fever (25/36). Anaemia was the most common (100\%) clinical feature followed by jaundice and splenomegaly (6/36). Aplastic anemia was the commonest cause that was observed in $27.78 \%$ (10/36) cases followed by Megaloblastic anemia $16.67 \%$ (6/36) and erythroid hyperplasia $13.88 \%$ (5/36).

Conclusion: Aplastic anaemia is leading cause of pancytopenia in this study followed by megaloblastic anaemia and hypersplenism being second and third common causes respectively. Pancytopenia should be suspected on clinical grounds when a patient presents with unexplained anemia, prolonged fever and tendency to bleed.
\end{abstract}

J Dhaka Med Coll. 2017; 26(2): 157-161

\section{Introduction}

Pancytopenia is a triad of simultaneous presence of anemia, leucopenia and thrombocytopenia. It is not a disease but findings that may result from a number of disease processes. Therefore it exists when Hemoglobin $(\mathrm{Hb})$ is less then $13.5 \mathrm{~g} / \mathrm{dl}$ in males or $11.5 \mathrm{~g} / \mathrm{dl}$ in females; the leucocytes count is less then $4 \times 10^{3} / 1$ and the platelets count is less than $150 \times 10^{3} / 1 .^{1}$

Most of the presenting symptoms are usually attributable to anemia or thrombocytopenia;
Leucopenia is an uncommon cause in the initial presentation but can become the most serious threat to life during the course of the disorder. The incidence of various disorders causing pancytopenia varies due to geographical distribution and genetic disturbances. ${ }^{2}$

Both hematopoietic and nonhematopoietic conditions manifest with features of pancytopenia. The underlying mechanisms are: decrease in hematopoietic cell production, marrow replacement by abnormal cells,

1. Dr. Mohammad Zaid Hossain, Associate Professor, Department of Medicine, Dhaka Medical College

2. Mohammad Manirul Islam, Assistant Professor, Department of Hematology, Dhaka Medical College

3. Major (Dr) Masuma Ahmed Salsabil, Classified Specialist in Pathology, Armed Forces Institute of Pathology, Dhaka Cantt

4. Dr. Quadrat-E-Elahi, Medicine Specialist, CMH, Dhaka

5. Dr. Mazharul Islam, EMO, Kurmitola General Hospital, Dhaka

6. Dr. Md. Abdullah Al Imran, Indoor Medical Officer, Department of Medicine, Dhaka Medical College Hospital, Dhaka

7. Dr. Pratyay Hasan, Indoor Medical Officer, Department of Medicine, Dhaka Medical College Hospital, Dhaka

8. Dr. Afsana Rahman, Resident Physician, Department of Medicine, Delta Medical College, Dhaka

9. Dr. Nira Ferdous, Consultant MOALLRC.

10. Dr. Israt Zerin Eva, Medical Officer, Department of Medicine, Dhaka Medical College, Dhaka

11. Prof. Khan Abul Kalam Azad, Professor, Department of Medicine, Dhaka Medical College, Dhaka

Received: 01 August $2017 \quad$ Revision: 20 August 2017

DOI: http://dx.doi.org/10.3329/jdmc.v26i2.38835

Accepted: 01 September 2017 
suppression of marrow growth and differentiation, ineffective hematopoiesis with cell death, defective cell formation which are removed from the circulation, antibody mediated sequestration or destruction of cells and trapping of cells in a hypertrophied and over active reticuloendothelial system. ${ }^{3,4}$

The workup of new-onset pancytopenia is extensive and should include a detailed clinical, medication, recreational drug, and environmental exposure history. Although bone marrow examination often reveals an underlying condition causing pancytopenia, it is not always conclusive. Understanding the various disorders that may cause pancytopenia can aid in the recommendation of additional testing and clinical evaluation when the marrow studies are not specific for a single etiology. Bone marrow examination play key role in evaluation of Pancytopenia. ${ }^{5}$ This allows complete assessment of marrow architecture and the pattern of distribution of any abnormal infiltrate and for the detection of focal bone marrow lesions. ${ }^{6,7}$ While bone marrow failure syndromes and malignancies are important causes, certain non-malignant conditions such as infection and nutritional anemia are equally important causes. ${ }^{5}$ The most common causes leading to pancytopenia on Bone Marrow examination are aplastic anaemia $(29.05 \%)$, Megaloblastic anemia (MA) (23.64\%), Hematological malignancies i.e. Acute Myeloid Leukemia (AML) (21.62\%), and erythroid hyperplasia (EH) $(19.6 \%) .{ }^{8}$

\section{Methods}

This was a cross-sectional study carried out in the Department of Medicine, Dhaka Medical College Hospital between December 2016 to July 2017. We included adult patients of both sexes having age 16 years and above. Criteria for inclusion were persistent pancytopenia on peripheral blood film of more than one week duration. Patients who were diagnosed cases of Malignancy, aplastic anaemia or Bleeding Disorder, cases of decompensated chronic liver disease, genetic causes of pancytopenia and pregnant females with pancytopenia were excluded. A written informed consent was obtained from all the patients after having fully explained the purpose and protocols of the study as well as risk to the patients. All patients underwent a detailed medical history and full physical examination followed by blood sampling for the investigations i.e. complete blood count with peripheral film, erythrocyte sedimentation rate (ESR), malarial parasites (MP), liver function test, renal function tests, $\mathrm{PT}$ and viral profile (HBsAg, Anti-HCV), ultrasonography of abdomen. After taking all the aseptic measures and with standard technique the diagnostic bone marrow aspiration and trephine biopsy were done from posterior iliac crest under adequate local anesthesia by using Salah and Jamshidi needles, respectively.

\section{Results}

There were 36 patients with pancytopenia which were included in this study. There were 20 (56\%) males and 16 (44\%) females with a 1.25:1 male to female ratio and a mean age 47.30 years \pm 15.01 SD.

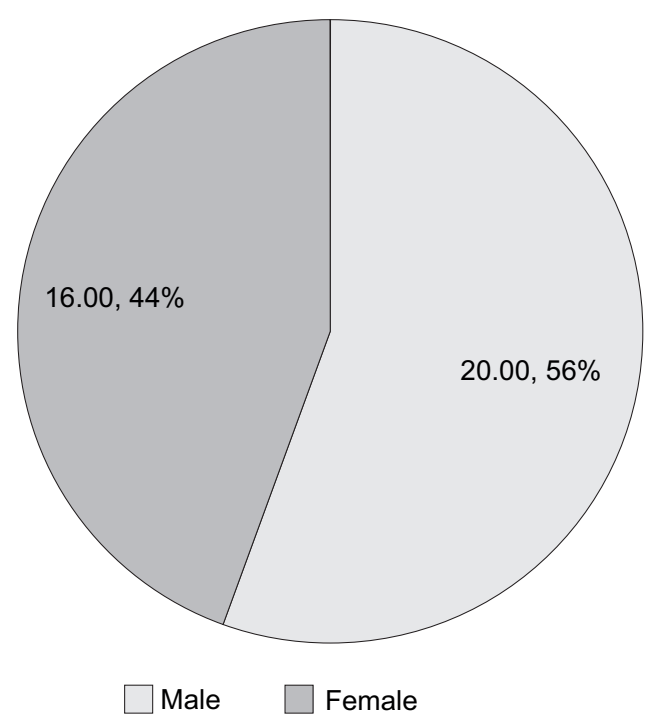

Fig.-1: Gender distribution of pancytopenic cases.

Among them 10 cases were factory worker, followed by hose wife (6/6), farmer $(5 / 36)$ 


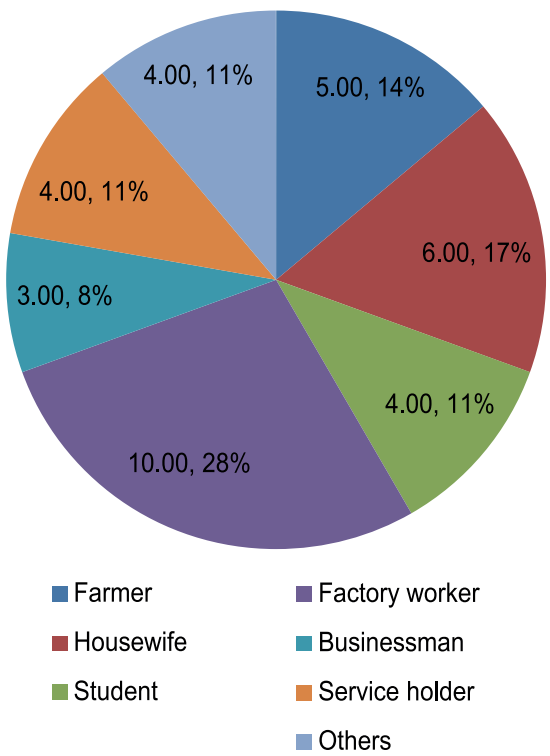

Fig.-2: Occupation distribution of pancytopenic cases.

The most common complaints were bleeding manifestation (29/36), followed by generalized weakness $(27 / 36)$ and fever (25/36).

It was found that anaemia (100\%) was the most common clinical feature followed by jaundice and splenomegaly $(6 / 36)$.

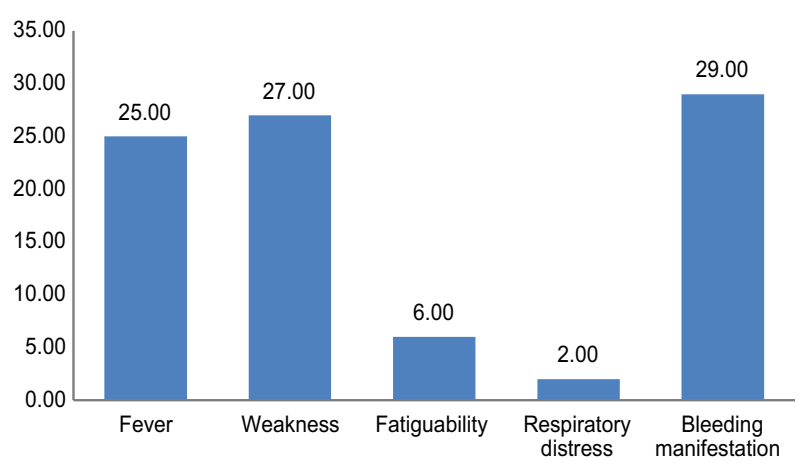

Figu.-3: Symptoms of pancytopenic cases.

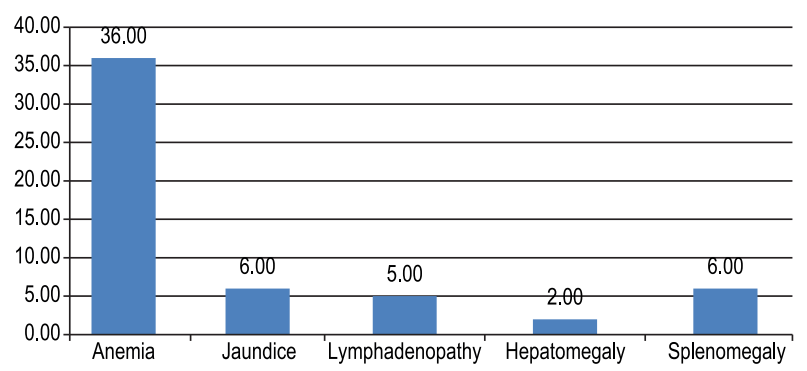

Fig.-4: Clinical feature of pancytopenic cases.

Aplastic anemia was the commonest cause that was observed in $27.78 \%(10 / 36)$ cases followed by Megaloblastic anemia $16.67 \%(6 / 36)$ and erythroid hyperplasia $13.88 \%(5 / 36)$.

Table-I

Frequency of age groups and causes of pancytopenia.

\begin{tabular}{lccccccccc}
\hline Age & Total & MDS & MA & AML & ALL & AA & SLE & RA & EH \\
group & $(\%)$ & $(\%)$ & $(\%)$ & $(\%)$ & $(\%)$ & $(\%)$ & $(\%)$ & $(\%)$ & $(\%)$ \\
\hline $16-25$ & 6.00 & 0.00 & 0.00 & 0.00 & 1.00 & 4.00 & 0.00 & 0.00 & 1.00 \\
& $(16.67)$ & $(0.00)$ & $(0.00)$ & $(0.00)$ & $(2.78)$ & $(11.11)$ & $(0.00)$ & $(0.00)$ & $(2.78)$ \\
$26-35$ & 9.00 & 0.00 & 0.00 & 1.00 & 1.00 & 3.00 & 2.00 & 1.00 & 1.00 \\
& $(25.00)$ & $(0.00)$ & $(0.00)$ & $(2.78)$ & $(2.78)$ & $(8.33)$ & $(5.56)$ & $(2.78)$ & $(2.78)$ \\
$36-45$ & 9.00 & 0.00 & 2.00 & 1.00 & 0.00 & 3.00 & 1.00 & 0.00 & 2.00 \\
& $(25.00)$ & $(0.00)$ & $(5.56)$ & $(2.78)$ & $(0.00)$ & $(8.33)$ & $(2.78)$ & $(0.00)$ & $(5.56)$ \\
$46-55$ & 7.00 & 0.00 & 3.00 & 1.00 & 1.00 & 3.00 & 0.00 & 1.00 & 1.00 \\
& $(19.44)$ & $(0.00)$ & $(8.33)$ & $(2.78)$ & $(2.78)$ & $(8.33)$ & $(0.00)$ & $(2.78)$ & $(2.78)$ \\
$56-65$ & 2.00 & 1.00 & 1.00 & 0.00 & 0.00 & 0.00 & 0.00 & 0.00 & 0.00 \\
& $(5.56)$ & $(2.78)$ & $(2.78)$ & $(0.00)$ & $(0.00)$ & $(0.00)$ & $(0.00)$ & $(0.00)$ & $(0.00)$ \\
$>65$ & 3.00 & 3.00 & 0.00 & 0.00 & 0.00 & 0.00 & 0.00 & 0.00 & 0.00 \\
& $(8.33)$ & $(8.33)$ & $(0.00)$ & $(0.00)$ & $(0.00)$ & $(0.00)$ & $(0.00)$ & $(0.00)$ & $(0.00)$ \\
\hline
\end{tabular}

$\mathrm{MDS}=$ Myelodisplastic disorder, M.A=Megaloblastic anaemia, $\mathrm{AML}=$ Acute Myeloid Leukemia, $\mathrm{AML}=\mathrm{Acute}$ Lymphoblastic Leukemia A.A= Aplastic anaemia, SLE= systemic Lupus Erythematosus, RA= Rheumatoid Arthritis E.H= Erythroid Hyperplasia 
The Common causes Leading to Pancytopenia at Tertiary Care Hospital

Hossain MZ et al

Table-II

Frequency of pancytopenia among both gender.

\begin{tabular}{|c|c|c|c|c|c|c|c|c|c|}
\hline & Total & MDS & MA & AML & ALL & AA & SLE & RA & $\mathrm{EH}$ \\
\hline Male & 20.00 & 3.00 & 3.00 & 2.00 & 2.00 & 6.00 & 0.00 & 1.00 & 3.00 \\
\hline Female & 16.00 & 1.00 & 3.00 & 1.00 & 1.00 & 4.00 & 3 & 1.00 & 2.00 \\
\hline
\end{tabular}

\section{Discussion}

Pancytopenia is a common haematological condition often encountered in day to day clinical practice. The evaluation of the cause of pancytopenia starts from history, physical examination and various laboratory investigations including basic hematological, biochemical, radiological, and histopathological investigation.

Presenting symptoms of pancytopenia may be attributable to anaemia, leucopenia, and/or thrombocytopenia. Anaemia may present with fatigue, breathlessness, and cardiac symptoms. Neutropenia may present with febrile illness due to increased susceptibility to infections. Patients with thrombocytopenia may present with mucocutaneous bleed or bruising. Aplastic anemia was considered the most common cause of pancytopenia (27.78\%) in our study . It differs from other studies where megaloblastic anemia is the common cause. ${ }^{1,9}$ The diagnosis of in our study was established by characteristic bone marrow findings. The high prevalence of nutritional anemia in India and northern region of Pakistan has been cited for the increased frequency of megaloblastic anemia. Among the nutritional anemias Vit B12 defciency is more prevalent than folate deficiency in Pakistan ${ }^{10}$. Studies from Tariq Aziz et al, ${ }^{11}$ Iqbal et $\mathrm{al}^{12}$, Qazi et al. ${ }^{13}$ showed Megaloblastic anemia was found to be the major cause of pancytopenia. Our study showed that peak incidence of aplastic anemia in middle age group (16 to 55 years) with male preponderance. Studies in Philippines ${ }^{14}$ and $\mathrm{Nepal}^{15}$ reported that males were affected with aplastic anemia much more frequently than females, which might be a result of higher incidence of occupational exposure to chemicals and of pesticides exposure as a common etiological agent for aplastic anemia in these countries. Megaloblastic anemia and erythroid hyperplasia are the second most
(16.67\%). Peak incidence of Megaloblastic anemia from 36 to 45 years with equal distribution of gender in our study. In contrast, Khanduri et al found in their study that the peak incidence was seen in the age group of $10-30$ years (48\% of patients) and there was a preponderance of women $(71 \%) .{ }^{16}$ This variation in the frequency of etiology and other features among the studies possible due to the broad spectrum of etiologies or disorders behind pancytopenia. Among the 5 cases of erythroid hyperplasia, two cases were Kala Azar and chronic liver disease each, one was malaria.

\section{Conclusion}

Pancytopenia is not a disease. It is a haematological feature of varying aetiology with slight male preponderance in our study. Aplastic anaemia is leading cause of pancytopenia in this study followed by megaloblastic anaemia and hypersplenism being second and third common causes respectively. Pancytopenia should be suspected on clinical grounds when a patient presents with unexplained anemia, prolonged fever and tendency to bleed. The present study concludes that detailed primary hematological investigations along with bone marrow aspiration in cytopenic patients are helpful for understanding the disease process; to diagnose, or to rule out the causes of, cytopenia; and in planning further investigations and management of cytopenic patients.

\section{References}

1. Khunger JM, Arulselvi S, Sharma U, Ranga S, Talib VH. Pancytopenia a clinicohaematological study of 200 cases.Indian J Pathol Microbiol. 2002;45:375379.

2. Habib-Ur-Rehaman; Md. Fazil, Faiz .Md. Khan: The etiology pattern of pancytopenia in children up to 15 yrs. Pak Armed forces Medical. Jr. 2003:63:183-187

3. Shimamura A, Alter BP. Inherited aplastic anemia syndrome. In: Greer JP, Foerster J, Rodgers GM, 
Paraskevas F, Gladet B, Arber DA, et al, editors. Wintrobe's clinical hematology. Philadelphia: Lippincott Williams \& Wilkins; 2008:1173-11261.

4. Jha A, Sayami G, Adhikari RC, Panta AD, Jha R. Bone marrow examination in cases of pancytopenia. J Nepal Med Assoc. 2008;47:12-17.

5. 5. Varma N, Dash S. A reappraisal of underlying pathology in adult patients presenting with pancytopenia. Trop Geogr Med. 1992;44:322-327.

6. Bone marrow examination: indication and technique. In: Anesoft, Foucar K, editors. Bone marrow pathology. Hong Kong: American Society Clinical Pathology; 2001:30-47.

7. Nanda A, Basu S, Marwaha N. Bone marrow trephine biopsy as an adjunct to bone marrow aspiration. J Assoc Physicians India. 2002;50:893-895.

8. Jha A, Sayami G, Adhikari RC, Panta AD, Jha R. Bone marrow examination in cases of pancytopenia. JNMA J Nepal Med Assoc. 2008;47:12-17.

9. Ishtiaq O, Baqai HZ, Anwer F, Hussain N. Patterns of pancytopenia patients in a general medical ward and a proposed diagnostic approach. J Ayub Med Coll Abbottabad. 2004;16:8-13
10. Modood-ul-Mannan, Anwar M, Saleem M, Wiqar A, Ahmad M. A study of serum vitamin B12 and folate levels in patients of megaloblastic anemia in northern Pakistan. J Pak Med Assoc. 1995;45:187-188.

11. Aziz T, Ali L, Ansari T, Liaquat HB, Shah S, Ara J. Pancytopenia: megaloblastic anemia is still the commonest cause. Pak J Med Sci. 2010;26:132-136.

12. Iqbal W, Hassan K, Ikram N, Nur S. Aetiological Breakup in 208 Cases of Pancytopenia. J Rawal Med Coll. 2001;5:7-10.

13. Qazi RA, Masood A. Diagnostic evaluation of Pancytopenia. J Rawal Med Coll 2002;6:30-33.

14. Santra G, Das BK. A cross-sectional study of the clinical profle and aetiological spectrum of pancytopenia in a tertiary care centre. Singapore Med J. 2010;51:806-812.

15. Bhatnagar SK, Chandra J, Narayan S, Sharma S, Singh V, Dutta AK. Pancytopenia in children: etiological profle. J Trop Pediatr. 2005;51:236-239.

16. Khanduri U, Sharma A. Megaloblastic anaemia: prevalence and causative factors. Natl Med J India. 2007;20:172-5. 